\title{
The direction of hemispheric asymmetries for object categorization at different levels of abstraction depends on the task
}

\author{
Tobias Studer*, Ronald Hübner \\ Universität Konstanz, Fachbereich Psychologie, Fach D29, D-78457 Konstanz, Germany
}

Accepted 17 January 2008

Available online 4 March 2008

\begin{abstract}
In this study hemispheric asymmetries for categorizing objects at the basic versus subordinate level of abstraction were investigated. As predictions derived from different theoretical approaches are contradictory and experimental evidence is inconclusive in this regard, we conducted two categorization experiments, where we contrasted two experimental paradigms. In the first experiment, subjects had to verify whether a word and a laterally presented picture matched or not. In the second experiment, subjects had to identify laterally presented pictures of animals either at the basic or subordinate level by pressing a corresponding response key. Whereas the first experiment revealed an advantage of the left hemisphere (LH) for categorizing objects at the basic level and of the right hemisphere (RH) for categorizing at the subordinate level, just the opposite brain asymmetry was found in the second experiment. As the stimuli were identical in both experiments, hemispheric asymmetries seem to be strongly task dependent.
\end{abstract}

(c) 2008 Elsevier Inc. All rights reserved.

Keywords: Hemispheric asymmetries; Levels of categorization; Global/local processing

\section{Introduction}

Each object we confront in our everyday life can usually be categorized at more than only one level of abstraction (Jolicoeur, Gluck, \& Kosslyn, 1984; Rosch, Mervis, Gray, Johnson, \& Boyes-Braem, 1976). In most situations, objects are classified at the so-called basic level (e.g. dog). Basic categories are perceptually the most distinctive compared to categories at other levels and contain sufficient information for most of our everyday interactions (Rosch et al., 1976). In some situations, however, a more specific classification at the subordinate level can be necessary, for instance, if one refers to a specific object among other exemplars of the same basic category (e.g. a Dalmatian among other kinds of dogs). It is assumed that categorizations at the basic and subordinate level mainly result from perceptual processing (Jolicoeur et al., 1984; Kosslyn,

\footnotetext{
${ }^{*}$ Corresponding author. Fax: +49 7531883017.

E-mail address: tobias.studer@uni-konstanz.de (T. Studer).
}

Alpert, \& Thompson, 1995; Tanaka, Luu, Weisbrod, \& Kiefer, 1999).

The main challenge for our cognitive system in this regard is to accomplish different classifications with identical visual input. For basic-level classification visual inputs of different instances have to be mapped to the same output category. In this case, a certain amount of information has to be ignored. For subordinate-level categorization, on the other hand, very similar visual inputs have to be sorted into different subordinate categories, i.e. even small differences in perceptual features have to be considered. How does our cognitive system fulfill these somewhat opposed demands?

Two major kinds of ideas have been proposed as a solution to this problem. One approach, linked to research on object categorization and object representation, focuses on the kinds of representations that are used for categorizations. The other approach, inspired by research on object perception, focuses on the perceptual features that might be differentially diagnostic for different kinds of categorizations. Both accounts also predict hemispheric asymmetries 
for categorizing visually presented objects at the basic or subordinate level, which are the focus of the present paper. Before we report our own experiments, both approaches and corresponding empirical research will now be considered in turn.

As one solution to the problem of categorizing objects at the basic or the subordinate level it has been proposed that there might be different kinds of representations for different categorization tasks located in different subsystems in the brain. One kind of representation could subserve classification at the basic, and another kind of representation classification at the subordinate level. Within this representation account it is further assumed that the cerebral hemispheres differ with respect to these representations (e.g. Laeng, Zarrinpar, \& Kosslyn, 2003; Marsolek, 1999). According to Marsolek (1999), for instance, each hemisphere has a bias toward storing the shape of objects in a specific representational format. The left hemisphere (LH) stores visual objects in an abstract format in the sense of a prototype. That is, those features are stored that are invariant over the exemplars of a category. These features of an object or object parts are represented independently of each other (Marsolek \& Burgund, 1997). Such a representational format should be helpful for categorizing objects at the basic level as a subset of features is always present in all exemplars of a basic category but often in different configurations. For instance, all kinds of pianos possess the feature 'keyboard' but this feature can be present in different configurations in an upright piano compared to a grand piano (c.f. Marsolek, 1999). The right hemisphere $(\mathrm{RH})$, on the other hand, is assumed to store specific exemplars. That is, object shapes are represented in a format that preserves the specific configuration of the features of a specific instance of a category. For instance, such a representation would include the information that the keyboard of a piano is arranged in line with the corpus of a grand piano but horizontally to that of an upright piano (c.f. Marsolek, 1999). Therefore, the specific representation of the RH can be used to differentiate between different subordinate categories.

Marsolek (e.g. 1999) provides some evidence for this representation account in perceptual priming experiments. In a priming phase line drawings of objects were presented centrally. In the following test phase line drawings of either the same objects or of other instances from the same basic categories were presented either in the left visual field (LVF; i.e. projected to the $\mathrm{RH}$ ) or the right visual field (RVF; i.e. projected to the $\mathrm{LH}$ ) for a very short time. The task for the subjects was to name these objects. Marsolek found that for stimuli presented in the RVF/LH performance was independent of whether the same exemplar had been used as prime or another exemplar of the same basic category. That is, priming was independent of the specific shape of an object. For LVF/RH-stimuli, in contrast, there was an advantage in performance when the prime and the test object were the same exemplar and a disadvantage in performance when the prime and test object were different exemplars of the same basic category. In this case, priming was sensitive to the specific shape of an object. This pattern of results could be produced with different stimulus material such as word forms (Marsolek, Kosslyn, \& Squire, 1992; Marsolek, Schacter, \& Nicholas, 1996; Marsolek, Squire, Kosslyn, \& Lulenski, 1994), pseudo-word forms (Burgund \& Marsolek, 1997), letterlike forms (Marsolek, 1995) and with line drawings of objects (Koutstaal et al., 2001; Marsolek, 1999; Saneyoshi, Kaminaga, \& Michimata, 2003). This indicates that the assumed representational systems are used for storing any kind of visual shapes, e.g. word forms as well as object shapes, and are therefore independent of specific semantic content (c.f. Marsolek, 1995).

The question is whether or not these findings could be transferred to object categorization at the basic and subordinate level. Therefore, Laeng et al. (2003) tried to replicate the findings from these priming experiments in a categorization experiment, using a picture-word verification task. They presented black-and-white drawings of objects (animals, artifacts as well as photos of faces of famous persons) either in the RVF/LH or in the LVF/RH. Directly after picture presentation a word which described the objects either at the basic level (e.g. 'dog') or at the subordinate level (e.g. 'collie') was presented acoustically through a loudspeaker. The task of the subjects was to indicate whether picture and word matched or not by pressing a corresponding response key. When subjects had to match basic category labels (e.g. 'dog') to pictures, responses were faster for RVF/LH-stimuli than for $\mathrm{LVF} / \mathrm{RH}$-stimuli. In contrast, when subjects had to match subordinate category labels (e.g. 'collie') to pictures, responses were faster for $\mathrm{LVF} / \mathrm{RH}$-stimuli than for RVF/LH-stimuli. Laeng et al. concluded from these observed visual field (VF)-effects that hemispheric asymmetries in categorizing objects at different levels of abstraction can be attributed to the fact that each hemisphere stores objects in a specific kind of representation which is in accordance with Marsolek's representation account (Marsolek, 1999).

Another solution to the problem of categorization at the basic and the subordinate level, which we call the feature account, assumes that specific features might be diagnostic for certain kinds of categorizations (e.g. Schyns, Bonnar, \& Gosselin, 2002). Hemispheric asymmetries should then be observable when the processing of these features is lateralized. In this regard, Schyns and colleagues (e.g. Schyns et al., 2002) have recently shown that stimuli need not necessarily be completely encoded and represented in a categorization task, but that task demands influence which features of an object are extracted and used to perform the task at hand (c.f. Schyns, 1998). It has been argued that the cognitive system might, therefore, rely on different aspects of the information that is present in the stimulus when having to categorize an object at the basic or the subordinate level (Archambault, O’Donnell, \& Schyns, 1999; Morrison \& Schyns, 2001; Schyns \& Oliva, 1997). At least for the domain of living things, there is some evidence that 
the global and local features of an object play a crucial role. For instance, early work of Eleanor Rosch (Rosch et al., 1976) has already shown that the overall shape of an object is diagnostic for categorizing at the basic level as categories at this level are most distinctive in this respect. For example, the global shape of a dog is quite distinct from the shape of a bird. Recent experimental work has confirmed this idea (Archambault, Gosselin, \& Schyns, 2000; Collin \& McMullen, 2005; Hayward, 1998; Large \& McMullen, 2006). In contrast, when an object has to be categorized at the subordinate level, then categorization must rely on local features, because objects of the same basic category are very similar in global shape (Archambault et al., 2000; Collin, 2006; Collin \& McMullen, 2005).

The involvement of global and local processing in object categorization also suggests that hemispheric asymmetries should be observable. Research on global/local processing has shown that the $\mathrm{RH}$ has a bias for processing the global features of an object, whereas the LH is biased toward the processing of local stimulus features. Corresponding asymmetries were found in lesion studies (e.g. Delis, Robertson, \& Efron, 1986; Robertson \& Lamb, 1991), electrophysiological studies (e.g. Heinze \& Münte, 1993; Malinowski, Hübner, Keil, \& Gruber, 2002; Proverbio, Minniti, \& Zani, 1998; Volberg \& Hübner, 2004), imaging studies (e.g. Fink et al., 1996; Heinze, Hinrichs, Scholz, Burchert, \& Mangun, 1998; Martinez et al., 1997) as well as response time studies (e.g. Hübner, 1997, 1998; Martin, 1979). For a recent review see Hübner and Volberg (2005).

The functional hemispheric asymmetries for global/local processing suggest that corresponding hemispheric differences should also be observable for categorizations at different levels of abstraction. The specific hemispheric asymmetry should occur depending on the level of the features that have to be processed. This feature account of hemispheric asymmetries for categorization should at least hold for the domain of living things. As the classification of these objects at the basic level predominantly relies on global stimulus features, the RH should be better in performing basic-level classification than the LH. In contrast, classification of objects at the subordinate level is mainly based on local features. Thus, subordinate-level classification should be better performed by the LH than by the RH.

To our knowledge, up to date there has been no attempt to test this feature account directly. However, there is some indirect evidence from studies of object recognition which usually corresponds to a classification at the basic level. If laterally presented objects have to be classified at the basic level, then, according to the feature account there should be a LVF/RH advantage for this task. Although older studies are inconclusive in this respect (e.g. Bryden \& Rainey, 1963; Levine \& Banich, 1982; Schmuller \& Goodman, 1980; Sergent \& Lorber, 1983; Wyke \& Ettlinger, 1961), and also exhibit a number of methodological problems (c.f. Biederman \& Cooper, 1991), more recent studies seem to support the feature account (e.g. Brooks \& Cooper, 2006; Koivisto \& Revonsuo, 2003; Laeng, Shah,
\& Kosslyn, 1999; McAuliffe \& Knowlton, 2000). An advantage of the LVF/RH was shown for the identification of laterally presented pictures of animals (Brooks \& Cooper, 2006; Laeng et al., 1999) and when subjects had to detect familiar objects with nonsense shapes as distracters (Koivisto \& Revonsuo, 2003; McAuliffe \& Knowlton, 2000). In contrast, when the object recognition task required an analysis of local details, an advantage for the RVF/LH has been observed (e.g. Koivisto \& Revonsuo, 2003; Laeng et al., 1999; Lag, Hveem, Ruud, \& Laeng, 2006; Laws \& Neve, 1999; Perani et al., 1995). Taken together, these results support the feature account.

As this short overview shows, there are two kinds of predictions about the pattern of hemispheric asymmetries that can be made for categorizing objects at the basic and at the subordinate level. Interestingly, at least for the domain of living things, where the global shape is an indicator for basic-level categorization and local features for subordinate-level categorization, the pattern of hemispheric asymmetries predicted by one approach is contrary to that predicted by the other. Whereas the feature account predicts that the RH and the LH have an advantage for categorizing objects at the basic and subordinate level, respectively, the representation account states exactly the opposite. The aim of the present study was to investigate the origin of the contradicting observations. Why is there evidence for both the feature and the representation account?

One conceivable reason could be that different experimental methods are applied in the two research areas. Research on categorization is traditionally based on experiments using word-picture verification tasks. That is, a word and a picture are presented and subjects have to indicate whether both match or not. This task seems to require to compare two object representations with respect to whether or not they are from the same (basic or subordinate) category. Therefore, functional hemispheric differences according to the representation account might be expected. On the other hand, research on global/local processing and object recognition prefers identification tasks. That is, a picture is presented and subjects have to identify it, e.g. by pressing a corresponding response key. In such a task, subjects are prepared in advance at which level the following object will have to be categorized. Therefore, they might prepare to look for certain diagnostic features that allow to categorize the shown object. There are, however, also differences in the employed stimulus sets. In global/local tasks, a limited number of stimuli is usually presented repeatedly whereas in categorization experiments a large stimulus set is employed where each stimulus is only shown once or at least only a few times. It is conceivable that subjects are able to extract diagnostic features only when they see the same stimuli repeatedly but not when each stimulus is shown only once.

To see whether these methodological differences account for the opposite results, we conducted two experiments in which the stimulus set was largely the same but the task 
for the observers varied. In the first experiment, we applied an experimental task similar to the one used by Laeng and colleagues (2003), i.e. the subjects had to match pictures and words. The aim was to examine whether Laeng et al.'s (2003) result can also be obtained with a stimulus set whose relatively small size corresponds to that usually used in global/local experiments. The results of this task were then compared to those of a second experiment, in which the same stimuli were used but where a more global/local like task was applied (e.g. Hübner \& Malinowski, 2002).

Our stimuli were shaded three-dimensional drawings of animals. In order to be able to clearly distinguish the predictions of the two approaches, we selected animals as stimuli which could be distinguished by their global shape at the basic level and by a local surface pattern at the subordinate level (for examples, see Fig. 1). That is, the membership to a given basic category was defined by the global object shape, whereas the membership to a given subordinate category was defined by a combination of global shape and local texture. If the feature account is valid, then there should be a LVF/RH advantage for categorizing our stimuli at the basic level and of the RVF/LH for categorizing them at the subordinate level. If, on the other hand, the representation account is valid, then the pattern of hemispheric asymmetries should be reversed.

\section{Experiment 1}

In our first experiment, we used a word-picture verification task similar to the one used by Laeng et al. (2003). The question was: Can the pattern of hemispheric asymmetries predicted by the representation account be obtained with a stimulus set that is smaller and that is clearly defined by global and local features with such a task? The subjects

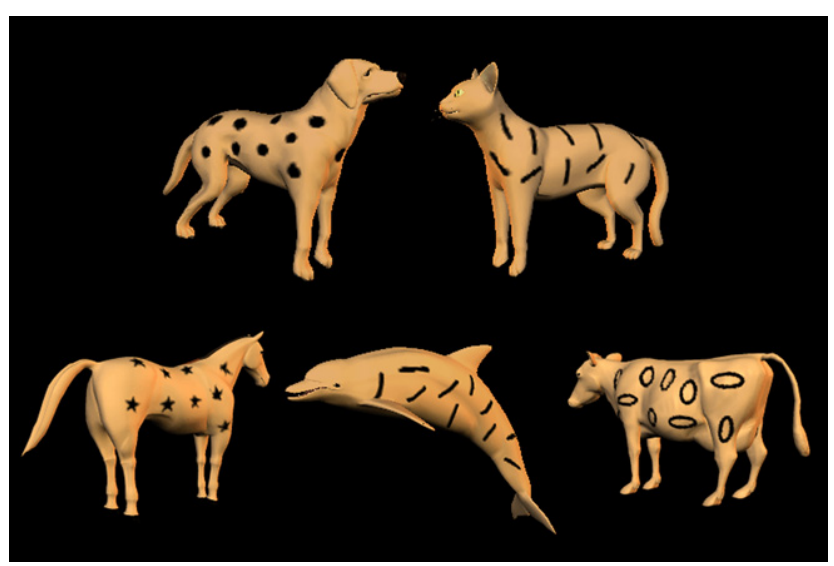

Fig. 1. Examples of stimuli used in all experiments. Basic categories were defined by animal shape (dog, cat, horse, dolphin, and cow), subordinate categories by a combination of animal shape and a specific texture (e.g. striped vs. dotted dog). Note that all animals could appear in two orientations and their mirror reflection and in combination with any of the four texture types (dots, stripes, stars and ovals). had to decide whether a visually presented word describing an object at the basic or at the subordinate level matched a subsequently presented picture or not. Contrary to Laeng et al. (2003), we used a word-picture and not a pictureword verification task, because presenting the word first and then the picture was more similar to the procedure applied in our identification task in Experiment 2, where the cue that indicated the level of categorization was also presented first. Moreover, we supposed that the pictureword order might produce a bias in favor of the representation account. If the picture is shown first, subjects always have to encode and represent any relevant aspect of the stimulus because they do not know in advance which picture level will be relevant for the subsequent verification task. The fact that all aspects of an object are encoded could induce a verification strategy that involves specific representations.

If the verification task involves the proposed representations (e.g. Laeng et al., 2003), then the basic-level and subordinate-level categorization should be better accomplished for stimuli presented in the RVF/LH and those presented in the $\mathrm{LVF} / \mathrm{RH}$-stimuli, respectively. If, on the other hand, the categorization is based on diagnostic global and local features, then the opposite pattern of hemispheric asymmetries should be observed.

Besides the classification at the basic and the subordinate level we also included a condition where the subjects had to verify only the local texture of the objects. With this condition we wanted to investigate the relation between the processing of local features and object categorization at the subordinate level. Although both the recognition of local patterns and the categorization at the subordinate level somehow involve local processing, there are also clear differences. Namely for the local pattern the analysis of the global form is not necessary and could be ignored whereas for subordinate categorization both the global form and the local patterns are necessary. So the question was: If according to the representation account, there were an advantage for stimuli presented in the LVF/RH for subordinate-level categorization, what kind of lateralization would be observable for the local level alone?

\subsection{Method}

\subsubsection{Subjects}

Forty-eight subjects ( 34 female, 14 male; mean age 23.7 years) participated in this experiment. All were righthanded by self-report, and had normal or corrected-to-normal vision.

\subsubsection{Apparatus}

The stimuli were presented on a $21^{\prime \prime}$-color-monitor (Sony) with a resolution of $1024 \times 768$ pixels and a refresh rate of $85 \mathrm{~Hz}$. A DOS-based $\mathrm{C}++$ computer program run on a personal computer (PC) served for controlling stimulus presentation and response registration. 


\subsubsection{Stimuli}

The stimuli used in the present experiments were designed with the computer software Curious Labs Poser 5. Three-dimensional images of cats, dogs, horses, cows, and dolphins in orange-brown color served as stimuli. The animals were additionally covered with a texture of dots, lines, stars, or ovals. Each animal was combined with all four texture types. Consequently, neither the shape nor the texture alone determined the subordinate category, but only the combination of animal shape and texture. Each animal was rendered in orientations of $60^{\circ}$ and $120^{\circ}$ in the depth plane and mirror images of each picture were made. Thus, there were four orientations of each animal stimulus (for examples of the stimuli, see Fig. 1). The stimuli were presented on a black background. The size of the stimuli depended both on the type of the animal and on its orientation. Height varied from $3.89^{\circ}$ to $4.62^{\circ}$, width from $4.69^{\circ}$ to $5.58^{\circ}$. The stimuli were presented at an eccentricity of $2.79^{\circ}$ (center of the screen to center of the stimulus).

For each subject there were four different basic categories (animals) and four subordinate categories resulting from a combination of two animal shapes and two textures. In order to avoid that the observed effects are just attributable to the use of special stimulus combinations, the basic and subordinate categories varied across subjects: one third of subjects had cats, dogs, horses, and dolphins as basic categories and striped cats, dotted cats, striped dogs, and dotted dogs as subordinate categories. Another third of subjects had the same basic categories, but horses and dolphins, each in combination with either stars or ovals as subordinate categories. The remaining 16 subjects had cats, dogs, horses, and cows as basic categories, and horses and cows, each either with dots or stripes as subcategories. So, each subject had four basic and four subordinate categories, but the specific stimuli differed between subjects.

\subsubsection{Procedure}

In this experiment, a word-picture verification task was used. Subjects were seated at a distance of $100 \mathrm{~cm}$ from the screen on which the stimuli appeared. A chin rest was used in order to keep the distance from the screen constant and to avoid head movements. Each trial started with the appearance of a short word, which named an object at the basic (e.g. "dog") or at the subordinate level (e.g. "Peppo" for the dog with dots), at the center of the screen for $300 \mathrm{~ms}$. The label served at the same time as fixation point and subjects were instructed to keep their eyes at this point during the whole experiment and were informed that it would make no sense to gaze to the left or to the right as stimuli would appear with equal probability in both visual fields. Additionally, there was a condition in which only the local texture had to be classified, irrespective of the animal on which it appeared. Then a stimulus occurred either in the LVF or RVF for $93 \mathrm{~ms}$ and the subjects had to indicate whether the label named the picture correctly or not by pressing a corresponding response button. The mapping of 'yes' and 'no' to the buttons was balanced across sub- jects as well as response hand. Response times were measured from the beginning of stimulus presentation. Thousand milliseconds after the response the next label appeared.

Three factors were varied in this experiment: level of categorization (basic or subordinate level, or local patterns), visual field ( $\mathrm{LVF} / \mathrm{RH}$, or $\mathrm{RVF} / \mathrm{LH}$ ), and response (same or different). After one practice block of 96 trials, 8 blocks of 96 trials were run resulting in 64 observations per condition and subject.

\subsection{Results}

Median latencies and mean error rates for 'same'responses and 'different'-responses were computed separately. For each type of responses, analyses of variance were conducted for both response times and error rates, respectively, with level of categorization, and visual field as repeated measures variables. 'Different'-responses were analyzed separately, because only with 'same'-responses one can be sure that a complete categorization at the required level has taken place. 'Different'-responses can be given for multiple reasons and are also dependent on the specific kind of distracter. Nonetheless, results of different responses are reported for the sake of completeness, and because they allow other interesting insights into stimulus categorizations in matching tasks. The additional condition of matching only the local textures was also analyzed separately.

\subsection{1. 'Same'-responses}

2.2.1.1. Response times. Averaged across visual field, verifications at the basic level ( $466 \mathrm{~ms}$ ) were faster than verifications at the subordinate level $(615 \mathrm{~ms}), F(1,47)=23.02$, $p<.001$.

Most important, we found a significant two-way interaction between the factors level of categorization and visual field, $F(1,47)=5.76, p<.05$ (see Fig. 2). Basic-level judgments were faster $(13 \mathrm{~ms})$ for $\mathrm{RVF} / \mathrm{LH}$-stimuli than for LVF/RH-stimuli, whereas subordinate-level judgments were faster $(19 \mathrm{~ms})$ for $\mathrm{LVF} / \mathrm{RH}$-stimuli than for RVF/ LH-stimuli. Planned comparisons showed that the VFeffect for matching at the basic level just failed shortly to reach significance, $t(47)=1.44, p=.079$, as did the VFeffect for matching at the subordinate level, $t(47)=1.64$, $p=.055^{1}$

The data for the local condition in which only the local textures had to be verified, were analyzed separately. Responses to the local patterns were faster $(16 \mathrm{~ms})$ for $\mathrm{RVF} / \mathrm{LH}$-stimuli than for LVF/RH-stimuli, $t(1,47)=$ $1.75, p<.05$.

\footnotetext{
${ }^{1}$ The specific stimuli had no reliable effect on the appearance and direction of hemispheric asymmetries. The three-way interaction between the factors level of categorization, visual field and stimulus type was not significant, neither in response times, $F(2,45)=1.55, p=.222$, nor in error rates, $F(2,45)=0.68, p=.510$.
} 


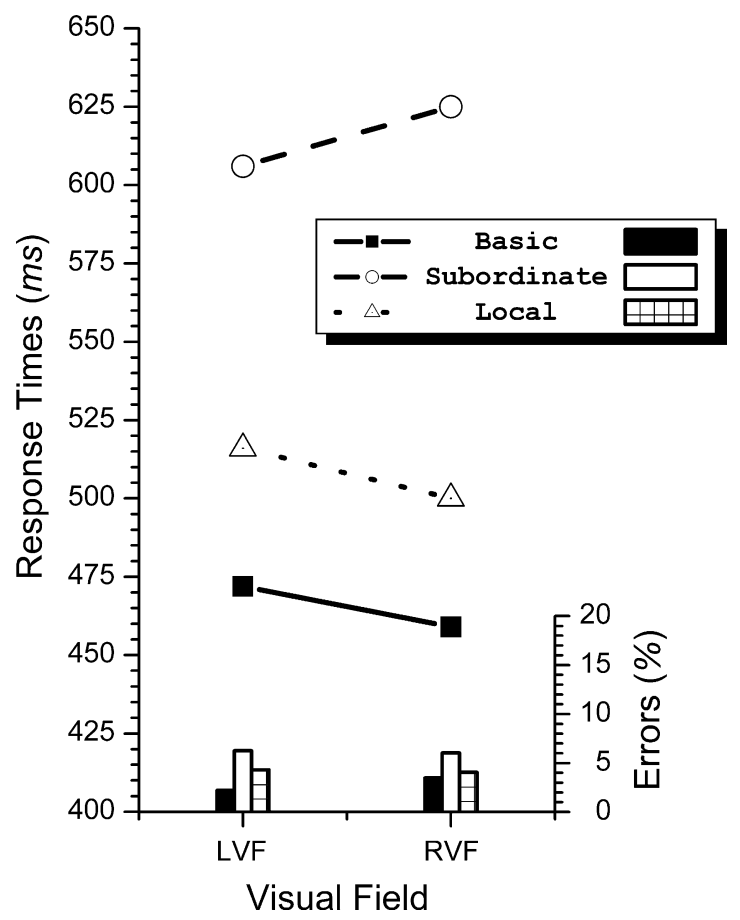

Fig. 2. Response times (lines) and error rates (bars) of the 'same'responses in Experiment 1: Categorizations at different levels of abstraction (basic level, subordinate level, and local patterns) plotted separately for presentations in the $\mathrm{LVF} / \mathrm{RH}$ and the $\mathrm{RVF} / \mathrm{LH}$.

2.2.1.2. Error rates. Errors occurred on average in $4.38 \%$ of the trials. Subjects generally made fewer errors for categorizations at the basic level $(2.84 \%)$ than for categorizations at the subordinate level $(6.14 \%), F(1,47)=23.30, p<.001$. We did not find an interaction between level of categorization and visual field. These results, however, rule out that the significant effects in response times might be due to a trade-off between speed and accuracy. Finally, there was no VF-effect for the local texture condition, either, $t(1,47)=.08, p=.41$.

\subsection{2. 'Different'-responses}

2.2.2.1. Response times. Averaged across visual field, correct rejections at the basic level $(528 \mathrm{~ms})$ were faster than those at the subordinate level (718 ms), $F(1,47)=31.25, p<.001$. Further, there was a significant two-way interaction between the factors level of categorization and visual field, $F(1,47)=5.23, \quad p<.05$ (see Fig. 3). Basic-level judgments were faster $(10 \mathrm{~ms})$ for RVF/LH-stimuli than for LVF/RH-stimuli, whereas subordinate-level judgments were faster $(39 \mathrm{~ms})$ for $\mathrm{LVF} /$ $\mathrm{RH}$-stimuli than for RVF/LH-stimuli. Planned comparisons showed that the VF-effect for rejecting at the basic level was significant, $t(47)=2.89, p<.05$, as was the VF-effect for rejecting at the subordinate level, $t(47)=3.43, p<.05$. For the local condition in which only the local textures had to be verified, responses were faster (16 ms) for RVF/LH-stimuli than for $\mathrm{LVF} / \mathrm{RH}$ stimuli, $t(1,47)=4.93, p<.05$.

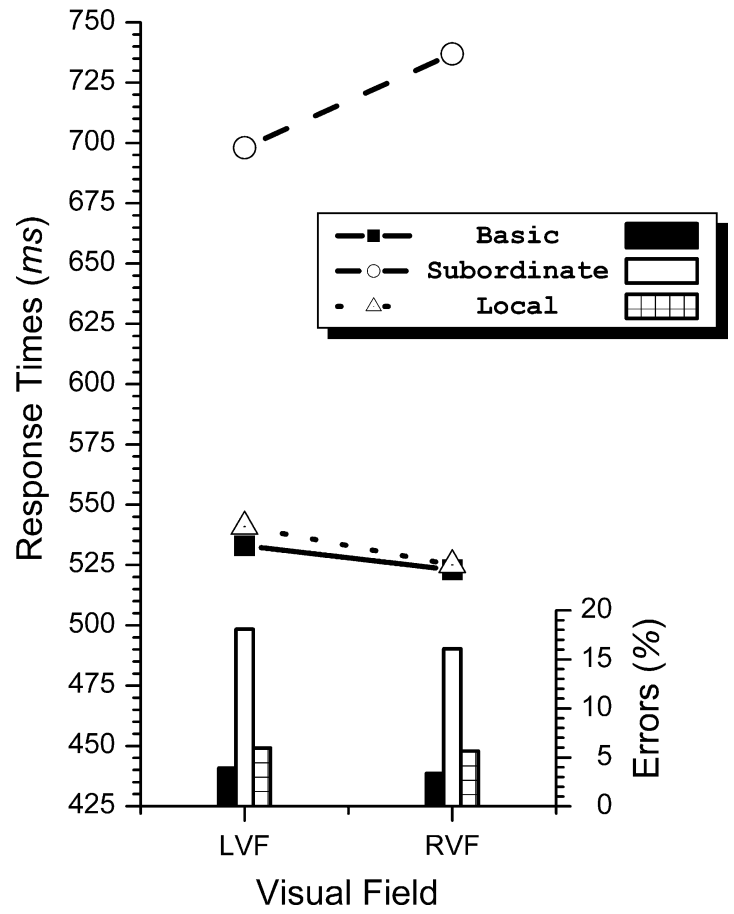

Fig. 3. Response times (lines) and error rates (bars) of the 'different'responses in Experiment 1: Categorizations at different levels of abstraction (basic level, subordinate level, and local patterns) plotted separately for presentations in the $\mathrm{LVF} / \mathrm{RH}$ and the $\mathrm{RVF} / \mathrm{LH}$.

2.2.2.2. Error rates. Errors occurred on average in $8.84 \%$ of the trials. Subjects generally made fewer errors for correct rejections at the basic level $(3.64 \%)$ than for those at the subordinate level $(17.07 \%), F(1,47)=117.81, p<.001$. We did not find an interaction between level of categorization and visual field, so that the significant effects in response times are not due to a trade-off between speed and accuracy. Finally, there was no VF-effect for the local texture condition, either, $t(1,47)=.08, p=.39$.

\subsubsection{Subanalysis of 'different'-responses at the subordinate level}

The present experiment was designed in a way that on 'different' trials for the subordinate categorization task the distracter always differed on one dimension (e.g. the global form was different) but overlapped on the other dimension (e.g. the local texture was the same). For instance, when the cue announced the presentation of the striped dog, the distracter could either be a striped cat (mismatch of the global form) or a dotted dog (mismatch of the local pattern). This design allows to differentiate between correct rejections that were based on the global form and those based on the local patterns. To see whether the level had any effect on the VF-effects, we further analyzed the 'different' data for the categorization at the subordinate level.

Median response times of correct rejections only at the subordinate level and error rates, respectively, were entered into a 2-factor ANOVA for repeated measurements on the 
factors mismatching feature (global form, or local pattern) and visual field (LVF/RH, or RVF/LH). Rejections were generally faster when the global form mismatched (693 $\mathrm{ms})$ than when the local pattern mismatched (781 ms), $F(1,47)=10.15, p<.01$. There was also a twoway interaction between the factors mismatching feature and visual field, $F(1,47)=4.65, p<.05$, indicating that a mismatch of the global form was detected faster for LVF/RH-stimuli than for RVF/LH-stimuli (39 ms), whereas a mismatch of the local pattern was detected faster for RVF/LH-stimuli compared to LVF/RH-stimuli (21 ms; see Fig. 4). Planned comparisons revealed that the VFeffect for the mismatch of the global form was significant, $t(47)=4.20, p<.05$, the VF-effect for mismatches of the local pattern, however, was not, $t(47)=.81, p=.19$. For error rates, we found a main effect for the factor mismatching feature, $F(1,47)=13.68, p<.01$, indicating that rejections based on the global form $(13.33 \%)$ were more accurately accomplished than those based on the local pattern $(20.13 \%)$. No other effects were significant.

\subsection{Discussion}

The aim of our first experiment was to show that hemispheric asymmetries as predicted by the representation account could be found with a small set of natural stimuli in a word-picture verification task similar to the one used by Laeng et al. (2003). For the main analysis, we included only trials that required a 'same' response, because a 'dif-

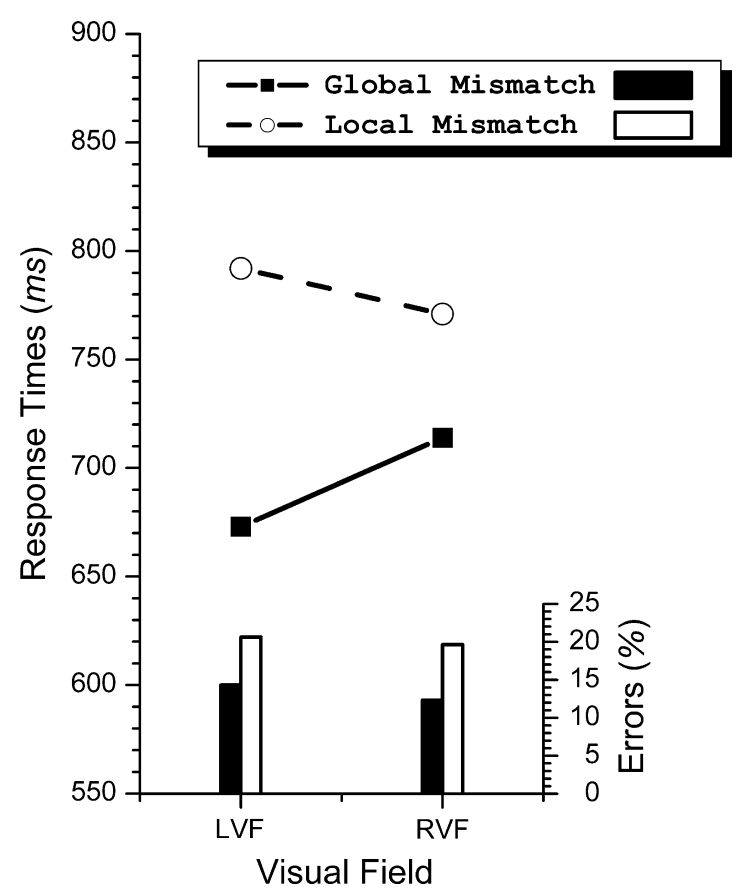

Fig. 4. Response times (lines) and error rates (bars) for the subanalysis of 'different' responses at the subordinate level in Experiment 1: Rejections at the subordinate-level dependent on the distracter type (i.e. either a mismatch of the global form or of the local pattern) plotted separately for presentations in the $\mathrm{LVF} / \mathrm{RH}$ and the $\mathrm{RVF} / \mathrm{LH}$ (see text for details). ferent' response does not necessarily correspond to a complete recognition or categorization of the distracter. In line with other research using a verification task (Jolicoeur et al., 1984; Laeng et al., 2003; Rosch et al., 1976) we found that verifications at the basic level were generally faster than those at the subordinate level. This suggests that more information has to be processed to categorize objects at the subordinate than at the basic level.

Concerning the hemispheric asymmetries, we found similar results as Laeng and colleagues (2003; see Fig. 2). Although the tests of the individual VF-effects failed shortly to reach significance, the interaction between level of categorization and VF was reliable and in the expected direction. There was a strong tendency that basic-level categorizations were better accomplished in the RVF/LH, and that subordinate-level categorizations were better accomplished in the LVF/RH. It should be noted that we found the same pattern of VF-effects as Laeng et al. (2003), even though we used a word-picture verification task and a smaller stimulus set. This is surprising, because some of our factors were quite specific and could also have favored the analysis of global and local features to perform the task. For instance, the stimuli were designed in a way, that it is clearly the global form that differentiates between basic categories, whereas the local patterns differentiate between subordinate members of a category. And even the fact that our subjects could prepare the processing of these relevant features in advance, as the label was always presented before the picture, did not prevent results in line with the representation account.

The analysis of 'different'-responses seems to reveal the same pattern of results (see Fig. 3). There was an advantage of the $\mathrm{RVF} / \mathrm{LH}$ for correct rejections at the basic level and an advantage of the $\mathrm{LVF} / \mathrm{RH}$ for correct rejections at the subordinate level. However, these results cannot readily be interpreted, because a correct rejection does not imply that the stimulus was completely identified at the required level. Rather, the rejection might have also been based on the type of distracter object. Indeed, if we consider our data in detail, then this suspicion is confirmed. The analysis of how the correct rejections of mismatches at the subordinate level depended on the specific mismatch information revealed that rejections based on global form differences were accomplished faster in the $\mathrm{LVF} / \mathrm{RH}$, whereas those based on local pattern differences were faster in the RVF/ LH. This result is in accord with the feature account. Moreover, verifying local patterns was better accomplished in the RVF/LH than in the LVF/RH, which also corresponds to the hemispheric asymmetry expected for local processing by the feature account.

Thus, a closer look at the data shows that the results for the 'different'-responses do less reflect the use of certain types of representations but rather seem to be the result of global or local processing. Although the overall analysis of the 'different' data seems to be in line with the prediction of the representation account (see upper curve in Fig. 3), it is merely the result of collapsing the data for the different 
distracter types (Fig. 4). The fact that the VF-effect for the global mismatch is larger than that for the local mismatch is responsible for the misleading overall result. At least, these considerations demonstrate that one should be careful with collapsing 'same' and 'different'-trials in a verification experiment.

Nevertheless, the analysis of the 'same' responses in Experiment 1 - which are the most unambiguous data with regard to categorization-supports the representation account. However, as mentioned in the Introduction, there is also some evidence that the feature account is valid. Our idea was, that task demands might be responsible for these contradictory findings. Therefore, in Experiment 2, we used the same stimulus set, but the subjects had to perform an identification task.

\section{Experiment 2}

The identification task used in this experiment was similar to that used in global/local studies. We expected that identification would lead to hemispheric asymmetries that differ from those in the previous experiment. According to the feature account, individual feature levels (global or local) are diagnostic for specific categorizations and, therefore, determine the direction of the hemispheric asymmetries. In particular, we hypothesized that the $\mathrm{RH}$ should have an advantage for categorizing objects at the basic level, whereas the LH should be specialized for categorizing objects at the subordinate level. In order to test these predictions, our subjects had to categorize laterally presented objects either at the basic or at the subordinate level by pressing a corresponding response key.

As in the word-picture verification experiment, we defined four categories at each level that had to be indicated by pressing one out of four response buttons, respectively. The use of response buttons, however, introduced an imbalance with respect to the number of response alternatives in comparison with the previous experiment, because only two response alternatives ('same' and 'different') are involved in the verification task, whereas four alternatives are involved in the identification task. Thus, in order to see whether or not the number of response alternatives, and, at the same time, the number of categories is crucial for the direction of hemispheric asymmetries, we included two conditions in the experiment. Half of the subjects had to identify four categories by pressing one out of four response buttons. In this condition we had the same number of categories as in the word-picture verification task in Experiment 1, but more response alternatives. The other half of the subjects had to identify only two basic categories and four exemplars mapped on two response buttons. Accordingly, in this condition we had the same number of response alternatives as in the word-picture verification task, but fewer categories. These conditions should allow to evaluate, at least to some extent, the effect of the number of categories and of response alternatives on hemispheric differences.
Moreover, in order to restrict the number of necessary response alternatives, this time subjects did not have to identify the local pattern alone.

\subsection{Method}

\subsubsection{Subjects}

Forty subjects (33 female, 7 male; mean age 23.2 years) participated in the experiment. All were right-handed by self-report, and had normal or corrected-to-normal vision.

\subsubsection{Apparatus and stimuli}

Apparatus and stimuli were the same as in Experiment 1. However, as it could be shown in the first experiment that the specific animals had no influence on the results, all subjects had the same categories. Subjects with four categories had cats, dogs, horses, and dolphins as basic categories and striped and dotted dogs as well as striped and dotted cats as subordinate categories. Subjects with only two categories had only cats and dogs as basic categories and striped and dotted dogs as well as striped and dotted cats as subordinate categories (for examples, see Fig. 1).

\subsubsection{Procedure}

The task was an identification task. Subjects had to classify the presented animal stimulus either at the basic level (e.g. dog) or at the subordinate level (e.g. striped dog) by pressing a corresponding response button.

Subjects were seated at a distance of $100 \mathrm{~cm}$ form the computer screen on which the stimuli were presented. A chin rest was used in order to keep viewing distance constant and to avoid head movements. Each trial started with the presentation of a letter as cue for $300 \mathrm{~ms}$ at the center of the screen. The letter served at the same time as fixation point and subjects were instructed to keep their eyes at this point during the whole experiment and were informed that it would make no sense to gaze to the left or to the right, as stimuli would appear with equal probability in both visual fields. The letter " $T$ " indicated that the following picture should be classified at the basic level, whereas the letter "E" signaled a classification at the subordinate level. After a blank screen of $500 \mathrm{~ms}$ the stimulus was presented for $93 \mathrm{~ms}$ either in the LVF or in the RVF. The subjects responded by pressing one out of four or two response buttons, respectively. Each button served as response for basic labels as well as subordinate labels at the same time.

For the subjects with four buttons, there were four basic (cat, dog, horse, and dolphin) and four subordinate categories (dotted cat, striped cat, dotted dog, and striped dog). Each basic category was mapped onto one of the four response buttons. Also each subordinate category was mapped onto one of the same four response buttons. There were two mappings of stimuli to response buttons which were counterbalanced across subjects: Half of the subjects responded with the left hand to cat and horse with the left and right finger, respectively, and with the right hand to dog and dolphin with the left and right finger, respectively 
(basic-level task). For the subordinate-level task, these subjects responded to striped dog and dotted cat with the left and right finger, respectively, and to striped dog and dotted cat with the left and right finger, respectively. For the other half of the subjects, the response hands were switched.

For the subjects with only two response buttons, there were only two basic categories (cat and dog), but also four subordinate categories (dotted cat, striped cat, dotted dog, and striped dog). For the subordinate categories, it was necessary to keep four categories, because with only two categories (e.g. dotted and striped cats), subjects could have completely ignored the global animal form and just respond to the local patterns. Thus, there was always one basic category, one subordinate cat category and one subordinate dog category mapped on one of the two response buttons. There were four mappings of stimuli to response buttons which were counterbalanced across subjects: In half of the subjects, cat was mapped onto the left button and dog on the right button, for the other half this mapping was reversed (basic-level task). Subordinate categories were mapped on response buttons so that always one striped and one dotted animal were mapped onto the same response button. Consequently, the dotted cat and the striped dog were mapped on one response button and the striped cat and the dotted dog on the other. Within this constraint, all possible combinations of basic and subordinate categories were possible. One half of the subjects responded with the left hand and the other half with the right hand.

Response times were measured beginning with the presentation of the stimulus. Thousand milliseconds after the response the next cue appeared.

Three factors were varied in this experiment: Level of categorization (basic or subordinate level), visual field ( $\mathrm{LVF} / \mathrm{RH}$, or $\mathrm{RVF} / \mathrm{LH}$ ) and the number of response alternatives (two or four). The first two factors were randomized within each block, whereas the number of response alternatives was varied between subjects. Altogether there were eight conditions. Twelve blocks of 64 trials were run for subjects with four categories, so that we obtained 192 observations per condition and subject. Eight blocks of 64 trials were run for subjects with two categories, so that we obtained 128 observations per condition and subject.

\subsection{Results}

The median latencies of correct responses and mean error rates, respectively, were entered into a 3-factor ANOVA with all involved factors.

\subsubsection{Response times}

Categorizations at the basic level $(772 \mathrm{~ms})$ were faster than those at the subordinate level (1039 ms), $F(1,38)=70.42, p<.001$. Further, subjects were generally faster when they had to decide between two response alternatives $(811 \mathrm{~ms})$ compared to four alternatives $(1000 \mathrm{~ms})$, $F(1,38)=7.63, p<.01$. Both factors, however, also interacted significantly, $F(1,38)=10.82, p<.01$. This was due to the fact that the reduction of response alternatives had a stronger effect on basic-level categories (294 ms) than on subordinate-level categories ( $84 \mathrm{~ms})$.

Finally and most important, there was a significant interaction between the two factors level of categorization and visual field, $F(1,38)=6.40, p<.05$. As can be seen in Fig. 5, categorizations at the basic level were faster (13 ms) when the stimuli were presented in the LVF/RH than when they occurred in the RVF/LH, whereas categorizations at the subordinate level were faster $(20 \mathrm{~ms})$ for RVF-stimuli than for LVF-stimuli. Planned comparisons revealed that the VF-effect for identifications at the basiclevel failed shortly to reach significance, $t(39)=1.93$, $p=.086$, but that the VF-effect for identifications at the subordinate level was significant, $t(39)=2.01, p<.05$. The VF-effect, however, did not depend on the number of response alternatives, as can be seen by the fact that the three-way interaction of the factors visual field, level of categorization and number of response alternatives was far from significance, $F(1,38)=0.33, p=.572 .^{2}$

\subsubsection{Error rates}

Errors occurred on average in $12.1 \%$ of the trials. Categorizations at the basic level $(10.18 \%)$ were more accurate than those at the subordinate level $(14.00 \%), F(1,38)=18.81$, $p<.001$. Further, subjects who had only two response alternatives generally made fewer errors $(8.51 \%)$ than subjects who had four response alternatives (15.68\%), $F(1,38)=21.67, p<.001$. These two factors also interacted significantly, $F(1,38)=6.33, p<.05$. This reflects the fact that the reduction of response alternatives had a stronger effect on categorizations at the basic level $(9.39 \%)$ than on categorizations at the subordinate level $(4.96 \%)$.

Most important, there was a significant interaction of the level of categorization and visual field, $F(1,38)=5.36$, $p<.05$. Categorizations at the basic level were more accurate for stimuli that were presented in the LVF/RH $(9.38 \%)$ compared to the RVF/LH (10.98\%). Categorizations at the subordinate level, on the other hand, were more accurate for RVF/LH-stimuli (13.70\%) compared to LVF/RH-stim-

\footnotetext{
${ }^{2}$ We conducted a post hoc analysis of the data including block as within variable in the main ANOVA because some studies have shown that lateralization of certain functions sometimes emerges only after a certain amount of practice (e.g. Sullivan \& McKeever, 1985) while other functions seem to disappear after practice (e.g. Cowin \& Hellige, 1994). The number and length of blocks, however, differed between experiments and the whole range of stimuli and conditions was completely presented only after four blocks of trials. Therefore, we compared the first four blocks to the last four blocks in each experiment. In Experiment 1, we neither found an effect of block on the VF-effect in response times, $F(1,47)=1.99, p=.16$, nor in error rates $F(1,47)=3.36, p=.07$. Also in Experiment 2, the threeway interaction between $V F$, level of categorization, and block was not significant, neither in response times, $F(1,39)=2.41, p=.13$, nor in error rates, $F(1,39)=2.08, p=.16$. As can be seen in the $p$-values, there seems to be a small tendency toward a change in hemispheric asymmetries over time. This, however, only reflects that the VF-effects have a slight tendency to get smaller over time which applies for both experiments. But there is clearly no indication of a change in the direction of the VF-effects.
} 


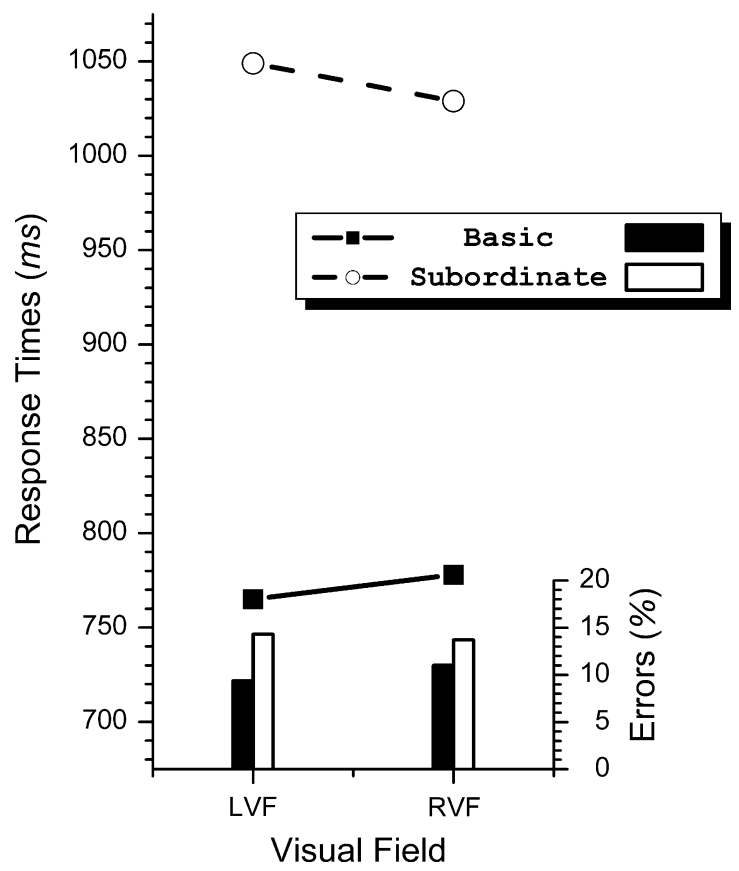

Fig. 5. Response times (lines) and error rates (bars) for categorizations at the basic and the subordinate level in Experiment 2 plotted separately for presentations in the LVF/RH and the RVF/LH.

uli $(14.30 \%)$. Planned comparisons revealed that the VFeffect for the identification at the basic level was significant, $t(39)=1.845, p<.05$, whereas the VF-effect for the identification at the subordinate level was not, $t(39)=1.09$, $p=.141$. The VF-effect, however, did not depend on the number of response alternatives, as can be seen by the fact that the three-way interaction of the factors visual field, level of categorization and number of response alternatives was far from significance, $F(1,38)=0.15, p=.704$. In sum, the analysis of the error rates mirrors the findings in response times.

\subsection{Comparison of Experiment 1 and 2}

In order to directly compare the results of the second to those of the first experiment, in particular concerning the direction of the VF-effects, we conducted an analysis including all subjects from Experiments 1 and 2 and including the variable task as between-subjects factor. The aim was to show that the direction of the VF-effects reliably differed between the two experiments and the respective tasks. Accordingly, the median latencies of subjects' response times of the 'same'-responses in Experiment 1 and the response times of the subjects in Experiment 2 were entered into a 3-factor ANOVA on the factors level of categorization, visual field, and task. We report only results that involve the factor 'task'.

The analysis first revealed that responses in the wordpicture verification task $(540 \mathrm{~ms})$ were significantly faster than those in the identification task (906 ms), $F(1,86)=78.33, p<.001$. The factor task also interacted significantly with the factor level of categorization,
$F(1,86)=6.19, p<.05$, indicating that the difference in response times between the two tasks was more pronounced for classifications at the subordinate level $(424 \mathrm{~ms})$ than for the classification at the basic level (307 ms). Most important, the three-way interaction between the factors level of categorization, visual field, and task was highly significant, $F(1,86)=11.95, p<.001$, indicating that the direction of the VF-effect was reversed depending on the specific categorization task. We also conducted planned comparisons separately for the basic and the subordinate level, which confirmed that both the simple VF-effect for the basic level, $F(1,86)=3.96, p<.05$, as well as the VF-effect for the subordinate level, $F(1,86)=6.19$, $p<.05$, differed significantly between the two tasks.

An analogue ANOVA was computed for the error rates which revealed that subjects were generally more accurate in the word-picture verification task than in the identification task, $F(1,86)=64.48, p<.001$. No other effects involving the task factor were significant.

\subsection{Discussion}

The aim of this experiment was to examine hemispheric asymmetries for categorizing objects at different levels of abstraction obtained with an identification task. Based on earlier global/local results our hypothesis was that the $\mathrm{RH}$ should be better at categorizing objects at the basic level, because this level of categorization should predominantly rely on the global features of a stimulus, whereas the $\mathrm{LH}$ is better in categorizing objects at the subordinate level, because this level should predominantly rely on local details. First of all, and in agreement with other experiments in this area (e.g. Jolicoeur et al., 1984; Rosch et al., 1976; Tanaka et al., 1999), we found that classifications at the basic level were generally faster and more accurate than those at the subordinate level. This can be attributed to the fact that more visual information has to be processed when objects are classified at the subordinate compared to when they are classified at the basic level.

Concerning the hemispheric asymmetries, our results support, as expected, the feature account. Categorizations at the basic level were accomplished faster and more accurate when the stimuli were presented in the LVF/RH, whereas categorizations at the subordinate level were faster and more accurate when stimuli were presented in the RVF/LH. Planned comparisons revealed that the individual VF-effects were reliable either in response times (subordinate level) or in the error rates (basic level). Our results are in accord with other studies (e.g. Marsolek \& Burgund, 2003), where VFeffects were sometimes also observed in response times and sometimes in error rates, depending on how subjects adjust their speed-accuracy trade-off. In any case, the reliable interaction between VF and level of categorization indicates that the hemispheres are differently involved in categorizing objects at the basic or subordinate level.

Because identification tasks and matching tasks usually differ in the number of response alternatives, it is important 
to note that this factor did not affect the VF-effects. Fewer response alternatives merely led to generally faster and more accurate responses. Thus, it must be the task as such that is responsible. This is also confirmed by the direct comparison of the VF-effects in the two experiments. The significant difference further confirms that the specific task has a strong influence on the direction of hemispheric asymmetries.

\section{General discussion}

In the present study, two experiments were conducted in which we investigated hemispheric asymmetries for the categorization of natural objects (animals) at the basic level and at the subordinate level. In this context, we contrasted two theoretical approaches - one that is more related to research on object categorization and another one that is more related to research on object perception. Interestingly, both accounts predict hemispheric asymmetries for categorization, but in opposite direction.

The first account stems from research on object categorization and assumes that each hemisphere represents objects in a specific format in memory (Marsolek, 1999). According to this representation account the LH prefers to represent objects as prototypes, i.e. as a collection of independent features, whereas the RH stores objects as concrete exemplars, thereby preserving the special configuration of the features of an object. Consequently, the representations of the $\mathrm{LH}$ are more useful for classifications at the basic level, whereas the representations of the $\mathrm{RH}$ are better suited for classifications at the subordinate level.

The second approach assumes that the pattern of hemispheric asymmetries directly results from processing differences of the hemispheres for global and local properties of a stimulus. Evidence suggests that, at least for living things, classification at the basic level relies on the global object forms, whereas differentiating objects at the subordinate level is achieved by also taking the local details of a stimulus into account (e.g. Archambault et al., 2000; Collin \& McMullen, 2005; Large \& McMullen, 2006). As the RH has a processing advantage for global aspects of a stimulus and the LH for local details (e.g. Hübner \& Volberg, 2005), it follows that the RH should classify objects more efficiently at the basic level than the LH. In contrast, the LH should have an advantage for classification at the subordinate level. ${ }^{3}$

\footnotetext{
${ }^{3}$ It should be noted that both the representation account and the feature account are more or less related to other functional hemispheric asymmetries that have been discussed in the literature. For instance, the representation account is similar to the distinction between the representation of categorical and metric spatial information in the two hemispheres (e.g. Kosslyn et al., 1989). It is assumed that the LH forms categorical spatial representations and the $\mathrm{RH}$ coordinate representations. The feature account, on the other hand, is related to processing asymmetries for different spatial frequency scales. It has been proposed that the LH shows a processing advantage for high spatial frequencies whereas the $\mathrm{RH}$ has an advantage for the processing of low spatial frequencies (e.g. Sergent, 1982).
}

Obviously, the predicted pattern of hemispheric asymmetries of this feature account is exactly opposite to that of the representation account. As there is empirical evidence for both accounts the question was: How can this difference be explained? By considering the methodological details we noticed that different experimental paradigms are commonly used in the two fields. Not only do the stimuli differ but also the task. Whereas an identification task is usually required in object recognition experiments, a verification task is mostly used in object categorization experiments. Accordingly, our hypothesis was that the direction of the observed VF-effects depends on the task required from the subjects. To test whether this is indeed the case, we contrasted the two tasks in the present study, while keeping the stimuli nearly identical.

In our first experiment, a word-picture verification task was applied, whereas an identification task was used in Experiment 2. Our results confirm that the direction of the VF-effects indeed depends on the specific task. In Experiment 1, with the verification task, there was a RVF/LH advantage for classifying the presented objects at the basic level, and a LVF/RH advantage for classifying the same objects at the subordinate level. In Experiment 2, however, with the identification task, the opposite relation was found. Because the stimulus type was identical in both experiments, the specific categorization task must have determined the direction of the observed VF-effects.

Although the interaction between VF and level of categorization was significant in both experiments, the simple VF-effects, computed separately for the basic and subordinate level, were significant in the identification task (Experiment 2), but only marginally significant in the verification task (Experiment 1). Presumably, our specific stimulus set was responsible for this latter result. It should have been very obvious for the subjects that the category membership of our stimuli was clearly defined by the global form and the local patterns. Under these conditions, a subset of subjects might have used global and local information also in the verification task. It is even conceivable that this happened only in a small proportion of the trials. This could, however, have increased the variance and thereby weakened the statistical power. Nevertheless, the observed VFeffects pointed clearly in the expected direction and were of comparable size in both experiments. Moreover, the direct comparison of the results of the two experiments confirmed that the VF-effects clearly differed between the two tasks and this finding was also reliable for each level of categorization separately. Thus, the direction of the observed hemispheric asymmetries clearly differed between the specific categorization tasks.

The identification task and the verification task do not only differ with respect to the required judgment type, but also with respect to the number of response alternatives. The verification task always has two response alternatives, which is not necessarily the case for the identification task. As the performance decreased with the number of response alternatives, a difference in hemi- 
spheric asymmetries might be attributed to the difference in performance between the tasks. To assess whether this is indeed the case, we varied the number of response alternatives in the identification task. Some subjects had two response alternatives, as the subjects in the verification task, whereas others had four response alternatives. Although reducing the number of response alternatives reduced the response times and error rates, the pattern of VF-effects remained unaffected by the number of response alternatives. Although the responses were still faster in the verification task than in the identification task with two response alternatives, it is unlikely that this difference was responsible for the reversal of the VF-effects. Rather, our experimental evidence suggests that the difference in the direction of the VF-effects was the consequence of specific categorization strategies applied by the subjects in the two experiments. It seems that each task encouraged the subjects to use specific representations and processes to accomplish the required categorizations. Thus, it seems that both the feature and the representation account are correct. Depending on the task, the one or the other approach can account for the corresponding VF-effects.

The question of why the subjects based their performance on different representations and processes and how these components are related to the cerebral hemispheres remain an objective of future research. However, some clues about these relations can already be extracted from the available literature on categorization. It has been shown that multiple systems are involved in object categorization, and that different categorization tasks involve different systems (e.g. Ashby \& Ell, 2001; Ashby \& Maddox, 2005; Ashby \& O’Brien, 2005; Koenig et al., 2005). Further, it has been demonstrated that different categorization strategies can be applied to the same categories, that these strategies can differ between subjects in the same experiment, and that even one and the same subject can use different strategies within one experiment (Koenig et al., 2005; Smith, Patalano, \& Jonides, 1998). Therefore, it is conceivable that word-picture verification tasks encourage or even require different representations and processes than identification tasks, which is then indicated by corresponding differences in the VF-effects.

One helpful differentiation in this regard is that between rule-based and similarity-based categorization (Koenig et al., 2005; Smith et al., 1998). In the first case, stimuli are categorized by exact and verbalizable rules (e.g. if it is a dog and if it is striped then press the right button). That is, subjects selectively attend to isolated features and decide whether these features are present or absent, and the results of these individual tests are then combined to select a response. In the second case, it is assumed that stimuli are represented in an integrated way and are matched against corresponding memory representations (prototypes and/or exemplars). In this case a decision is not made for each individual feature but for the stimulus as a whole (Koenig et al., 2005; Smith et al., 1998).
We suppose that in an identification task subjects base their categorizations on rules concerning the global and local stimulus features. That is, they separately judge the global form and the local textures, and then combine the results to test whether the features indeed comply with the rule. Consequently, VF-effects appear in correspondence with the relevant feature which has to be analyzed to solve the categorization task. In contrast, in the wordpicture verification task, the subjects presumably encode the stimuli as a whole and match the result to prototypes in the LH or to exemplars in the $\mathrm{RH}$, depending on the categorization level. Consequently, the VF-effects correspond to the kind of representation with which the stimulus was matched.

There are some reasons to believe that these different strategies were in fact applied in our experiments. First, rule-based categorization is a stage-like process, where features have to be identified individually, perhaps one after the other, and the results are then combined for deriving a decision. In contrast, similarity-based categorization is a parallel process, where similarity of the object with respect to different features is computed simultaneously (e.g. Smith et al., 1998). Consequently, rule-based categorization generally takes longer than similarity-based categorization (e.g. Allen \& Brooks, 1991; Smith \& Kemler, 1984). This difference is also evident in our data: Response times and error rates were increased in the identification task compared to the verification task. Moreover, this performance difference was more pronounced for subordinatelevel classifications. This might as well be attributed to a serial feature identification strategy in the identification task (c.f. Smith et al., 1998). The subjects first decided whether the stimulus is a cat or a dog and then whether the animal is striped or dotted. The results of the two decisions were then combined to derive an overall decision. Altogether, such a processing would take a relatively long time especially for categorizations at the subordinate level (c.f. Smith et al., 1998).

Second, Rouder and Ratcliff (2006) observed a relation between memory load and categorization strategy. They showed that the higher the degree of confusability the more a rule-based categorization strategy is used, because it requires less long-term memory capacity. In our identification task subjects always had to actively remember what the name of the stimulus was and which button corresponded to the correct response. In this case, it might have been more helpful to learn and use precise rules, how stimulus features and their combinations are mapped to the responses. Tasks like word-picture verification, on the other hand, per se do not require much long-term memory capacity, because the relevant category name is always presented together with the to be categorized stimulus. Additionally, a kind of semantic priming supports the decision process.

Third, multiple strategies of categorization that can be used by a subject even within a single experiment, can also explain the surprising result that, although in the verifica- 
tion task VF-effects generally pointed in the direction predicted by the representation account, in certain subtypes of this task there was also a VF-effect in accordance with the feature account. When the task was to detect a mismatch between the label and the stimulus at the subordinate level, a VF-effect in the direction as predicted by the feature account emerged, i.e. there was a LVF/RH advantage when the mismatch appeared at the global level, and a RVF/LH advantage when it appeared at the local level. In our proposed framework this suggests that in the word-picture verification task subjects always started by matching integrated representations. However, when they detected a certain amount of mismatch, then they switched to a more visually guided feature analysis to verify whether there was really a relevant mismatch and, if so, at which level it occurred (see Krueger, 1978, for a similar idea in perceptual matching tasks). That is, decisions about a mismatch are also based on decisions about the presence or absence of isolated global or local features.

Thus, our experiments show that there are multiple ways of representing and processing objects in the brain and that the hemispheres differ in this regard. However, one might ask: Why did the hemispheres evolve such biases in representations and processing strategies? Whereas traditional views of hemispheric differences emphasized that each hemisphere might fulfill different complex functions (e.g. dominance of the $\mathrm{LH}$ for verbal material and of the $\mathrm{RH}$ for visual material), modern approaches suggest that both hemispheres collaborate to produce one complex function (e.g. object recognition) but that each hemisphere contributes to this goal in a different way (c.f. Gazzaniga, Ivry, \& Mangun, 2002). As our experiments show, categorization and object recognition are not unitary functions and might sometimes even require contradictory operations. Therefore, it seems to be an evolutionary advantage to have different brain modules which can collaborate in a flexible and adaptive way to map the same visual input to different categories depending on the required task (c.f. Laeng et al., 2003; Marsolek \& Burgund, 1997).

One could argue that the conclusions that can be drawn from our study are limited by the fact that only animals were used as superordinate category. We used animals, because there is a considerable amount of evidence that global and local features are involved in categorizing these kinds of stimuli, whereas this is less clear for other categories. For our study, which focused on hemispheric asymmetries in object categorization, it was quite important to know which features are relevant for categorization in order to make clear predictions about the direction of hemispheric asymmetries according to the feature hypothesis. The aim of our study was to show that the specific task demands have an influence on categorization strategies and corresponding VF-effects and this could clearly be demonstrated. It is important to note, that our results also indicate that there are no VF-effects for categorization per se, but that they reflect the combination of underlying lateralized processes and representations. Thus, the effects become evident only under certain conditions. For instance, the feature account predicts that the direction of VF-effects depends on the object features that are relevant for the specific categorization. Therefore, the specific lateralization should be different when objects can clearly be differentiated by their local features, as has been supposed for some kinds of man-made tools. In this case, one would expect an advantage of RVF/LH-stimuli (e.g. Lag et al., 2006; Laws \& Neve, 1999; Perani et al., 1995).

Another limiting fact might be that, although we tried to design our stimuli so that they appeared quite 'natural', there was a difference between our basic level categories and our subordinate-level categories. The basic categories (dog, cat, etc.) were probably familiar to the subjects, whereas the subordinate categories (striped dog, dotted cat, etc.) were more artificial and had to be learned. We think, however, that this difference was not crucial for the purpose of our study. We wanted to investigate whether the task affects the direction of hemispheric asymmetries. As in both experiments basic-level categories were familiar and subordinate categories had to be learned, familiarity could not have produced the opposite results, because the direction of VF-effects changed for both levels. Moreover, we reproduced the same pattern of VF-effects as Laeng and colleagues (2003) who used a stimulus set which contained only pictures of familiar objects and a large number of objects and which were presented only a few times. As they found similar results as in our Experiment 1 we conclude that familiarity and number of stimuli did not play a crucial role for our findings.

Altogether, our experiments suggest that the specific classification task subjects have to accomplish determines the direction of observed VF-effects. The different tasks probably encouraged the subjects to use different strategies of categorization which involved specific processes and representations. An unconstrained identification of pictures of animals seems to favor the manifestation of hemispheric asymmetries based on the diagnostic features of an object, whereas matching of a label to a corresponding picture favors the manifestation of asymmetries which are predicted by an account that assumes different modes of storing objects in the two hemispheres. Thus, our results can be interpreted in the sense that there are no general hemispheric differences for the categorization at different levels of abstraction, but that the hemispheric differences reflect basic processing and representation asymmetries which are involved in the categorization at different levels. Therefore, when investigating processes of categorization, it should be taken into account which task and what kinds of stimuli are used.

\section{Acknowledgments}

We are grateful to Stephen Christman and four anonymous reviewers for helpful comments on an earlier version of this paper. We also thank Patrick Kleiner, Maddalena Brunetti, and Jan Schlösser for collecting the data. 


\section{References}

Allen, S. W., \& Brooks, L. R. (1991). Specializing the operation of an explicit rule. Journal of Experimental Psychology: General, 120(1), 3-19.

Archambault, A., Gosselin, F., \& Schyns, P. G. (2000). A natural bias for the basic level?. In L. R. Gleitman \& A. K. Joshi (Eds.) Proceedings of the twenty-second annual conference of the cognitive science society. $\mathrm{NJ}$ : Erlbaum.

Archambault, A., O’Donnell, C., \& Schyns, P. G. (1999). Blind to object changes: When learning the same object at different levels of categorization modifies its perception. Psychological Science, 10(3), 249-255.

Ashby, F. G., \& Ell, S. W. (2001). The neurobiology of human category learning. Trends in Cognitive Sciences, 5(5), 204-210.

Ashby, F. G., \& Maddox, W. T. (2005). Human category learning. Annual Review of Psychology, 56, 149-178.

Ashby, F. G., \& O’Brien, J. B. (2005). Category learning and multiple memory systems. Trends in Cognitive Sciences, 9(2).

Biederman, I., \& Cooper, E. E. (1991). Object recognition and laterality: Null effects. Neuropsychologia, 29, 685-694.

Brooks, B. E., \& Cooper, E. E. (2006). What types of visual recognition tasks are mediated by the neural subsystem that subserves face recognition? Journal of Experimental Psychology: Learning Memory and Cognition, 32(4), 684-698.

Bryden, M. P., \& Rainey, C. A. (1963). Left-right differences in tachistoscopic recognition. Journal of Experimental Psychology, 66, 568-571.

Burgund, E. D., \& Marsolek, C. J. (1997). Letter-case specific priming in the right cerebral hemisphere with a form specific perceptual identification task. Brain and Cognition, 35, 239-258.

Collin, C. A. (2006). Spatial-frequency thresholds for object categorisation at basic and subordinate levels. Perception, 35, 41-52.

Collin, C. A., \& McMullen, P. A. (2005). Subordinate-level categorization relies on high spatial frequencies to a greater degree than basic-level categorization. Perception \& Psychophysics, 67(2), 354-364.

Cowin, E. L., \& Hellige, J. B. (1994). Categorical versus coordinate spatial processing: Effects of blurring and hemispheric asymmetry. Journal of Cognitive Neuroscience, 6(2), 156-164.

Delis, D. C., Robertson, L. C., \& Efron, R. (1986). Hemispheric specialization of memory for visual hierarchical stimuli. Neuropsychologia, 24, 205-214.

Fink, G. R., Halligan, P. W., Marshall, J. C., Frith, C. D., Frackowiak, R. S. J., \& Dolan, R. J. (1996). Where in the brain does visual attention select the forest and the trees? Nature, 382, 626-628.

Gazzaniga, M. S., Ivry, R. B., \& Mangun, G. R. (2002). Cognitive neuroscience. The biology of the mind (2nd ed.). New York: W.W. Norton \& Company.

Hayward, G. H. (1998). Effects of outline shape in object recognition. Journal of Experimental Psychology: Human Perception and Performance, 24(2), 427-440.

Heinze, H. J., Hinrichs, H., Scholz, M., Burchert, W., \& Mangun, G. R. (1998). Neural mechanisms of global and local processing: A combined PET and ERP study. Journal of Cognitive Neuroscience 10, 485-498

Heinze, H.-J., \& Münte, T. F. (1993). Electrophysiological correlates of hierarchical stimulus processing: Dissociation between onset and later stages of global and local target processing. Neuropsychologia, 31(8), $841-852$

Hübner, R. (1997). The effect of spatial frequency on global precedence and hemispheric differences. Perception \& Psychophysics, 59 187-201.

Hübner, R. (1998). Hemispheric differences in global/local processing revealed by same-different judgements. Visual Cognition, 5, 457-478.

Hübner, R., \& Malinowski, P. (2002). The effect of response competition on functional hemispheric asymmetries for global/local processing. Perception \& Psychophysics, 64, 1290-1300.
Hübner, R., \& Volberg, G. (2005). The integration of object levels and their content: A theory of global/local processing and related hemispheric differences. Journal of Experimental Psychology: Human Perception and Performance, 31(3), 520-541.

Jolicoeur, P., Gluck, M. A., \& Kosslyn, S. M. (1984). Pictures and names: Making the connection. Cognitive Psychology, 19, 31-53.

Koenig, P., Smith, E. E., Glosser, G., DeVita, C., Moore, P., McMillan, C., et al. (2005). The neural basis for novel semantic categorization. Neuroimage, 24, 369-383.

Koivisto, M., \& Revonsuo, A. (2003). Object recognition in the cerebral hemispheres revealed by visual field experiments. Laterality, $8(2)$, 135-153.

Kosslyn, S. M., Alpert, N. M., \& Thompson, W. L. (1995). Identifiying objects at different levels of hierarchy: A positron emission tomography study. Human Brain Mapping, 3, 107-132.

Kosslyn, S. M., Koenig, O., Barret, A., Cave, C. B., Tang, J., \& Gabrieli, J. D. E. (1989). Evidence for two types of spatial representations: Hemispheric specialization for categorical and coordinate relations. Journal of Experimental Psychology: Human Perception and Performance, 15, 723-735.

Koutstaal, W., Wagner, A. D., Rotte, M., Maril, A., Buckner, R. L., \& Schacter, D. L. (2001). Perceptual specificity in visual object priming Functional magnetic resonance imaging evidence for a laterality difference in fusiform cortex. Neuropsychologia, 39, 184-199.

Krueger, L. E. (1978). A theory of perceptual matching. Psychological Review, 85(4), 278-304.

Laeng, B., Shah, J., \& Kosslyn, S. (1999). Identifying objects in conventional and contorted poses: Contributions of hemispherespecific mechanisms. Cognition, 70, 53-85.

Laeng, B., Zarrinpar, A., \& Kosslyn, S. M. (2003). Do separate processes identify objects as exemplars versus members of basic-level categories? Evidence from hemispheric specialization. Brain and Cognition, 53 , $15-27$.

Lag, T., Hveem, K., Ruud, K. P. E., \& Laeng, B. (2006). The visual basis of category effects in object identification: Evidence from the visual hemifield paradigm. Brain and Cognition, 60, 1-10.

Large, M.-E., \& McMullen, P. A. (2006). Hierarchical attention in discriminating objects at different levels of specificity. Perception \& Psychophysics, 68(5), 845-860.

Laws, K. R., \& Neve, C. (1999). A 'normal' category-specific advantage for naming living things. Neuropsychologia, 37, 1263-1269.

Levine, C., \& Banich, M. T. (1982). Lateral asymmetries in the naming of words and corresponding line drawings. Brain and Language, 17, 34- 45 .

Malinowski, P., Hübner, R., Keil, A., \& Gruber, T. (2002). The influence of response competition on cerebral asymmetries for processing hierarchical stimuli revealed by ERP recordings. Experimental Brain Research, 144, 136-139.

Marsolek, C. J. (1995). Abstract visual form representations in the left cerebral hemisphere. Journal of Experimental Psychology: Human Perception and Performance, 21, 375-386.

Marsolek, C. J. (1999). Dissociable neural subsystems underlie abstract and specific object recognition. Psychological Science, 10(2), 111-118.

Marsolek, C. J., \& Burgund, E. D. (1997). Computational analyses and hemispheric asymmetries in visual-form recognition. In S. Christman (Ed.), Cerebral asymmetries in sensory and perceptual processing (pp. 125-158). Amsterdam: Elsevier.

Marsolek, C. J., \& Burgund, E. D. (2003). Visual recognition and priming of incomplete objects: The influence of stimulus and task demands. In J. S. Bowers \& C. J. Marsolek (Eds.), Rethinking implicit memory (pp. 139-156). New York, NJ: Oxford University Press.

Marsolek, C. J., Kosslyn, S. M., \& Squire, L. R. (1992). Form specific visual priming in the right cerebral hemisphere. Journal of Experimental Psychology: Learning Memory and Cognition, 18, 492-508.

Marsolek, C. J., Schacter, D. L., \& Nicholas, C. D. (1996). Form-specific visual priming for new associations in the right cerebral hemisphere. Memory and Cognition, 24, 539-556. 
Marsolek, C. J., Squire, L. R., Kosslyn, S. M., \& Lulenski, M. E. (1994). Form specific explicit and implicit memory in the right cerebral hemisphere. Neuropsychology, 8, 588-597.

Martin, M. (1979). Hemispheric specialization for global and local processing. Neuropsychologia, 17, 33-40.

Martinez, A., Moses, P., Frank, L., Buxton, R., Wong, E., \& Stiles, J. (1997). Hemispheric asymmetries in global and local processing: Evidence from fMRI. Neuroreport, 8, 1685-1689.

McAuliffe, S. P., \& Knowlton, B. J. (2000). Hemispheric differences in object identification. Brain and Cognition, 45, 119-128.

Morrison, D. J., \& Schyns, P. G. (2001). Usage of spatial scales for the categorization of faces, objects, and scenes. Psychonomic Bulletin and Review, 8(3), 454-469.

Perani, D., Cappa, S. F., Bettinardi, S. V., Bressi, S., Gorno-Tempini, M., Mattarese, M., et al. (1995). Different neural subsystems for the recognition of animals and man-made tools. Neuroreport, 6, 1637-1641.

Proverbio, A. M., Minniti, A., \& Zani, A. (1998). Electrophysiological evidence of a perceptual precedence of global vs. local visual information. Cognitive Brain Research, 6, 321-334.

Robertson, L. C., \& Lamb, M. R. (1991). Neuropsychological contributions to theories of part/whole organization. Cognitive Psychology, 23, 299-330.

Rosch, E., Mervis, C. B., Gray, W. D., Johnson, D. M., \& Boyes-Braem, P. (1976). Basic objects in natural categories. Cognitive Psychology, 8, $382-452$.

Rouder, J. N., \& Ratcliff, R. (2006). Comparing exemplar- and rule-based theories of categorization. Current Directions in Psychological Science, $15(1), 9-13$.

Saneyoshi, A., Kaminaga, T., \& Michimata, C. (2003). The neural basis of object recognition for between- and within-category levels-an fMRI study. Technical Report on Attention and Cognition, 29, 1-5.
Schmuller, J., \& Goodman, R. (1980). Bilateral tachistoscopic perception, handedness and laterality: II. Nonverbal stimuli. Brain and Language, $11,12-18$.

Schyns, P. G. (1998). Diagnostic recognition: Task constraints, object information, and their interactions. Cognition, 67, 147-179.

Schyns, P. G., Bonnar, L., \& Gosselin, F. (2002). Show me the features. Understanding recognition from the use of visual information. Psychological Science, 13(5), 402-409.

Schyns, P. G., \& Oliva, A. (1997). Flexible, diagnosticity-driven, rather than fixed, perceptually determined scale selection in scene and face recognition. Perception, 26, 1027-1038.

Sergent, J. (1982). The cerebral balance of power: Confrontation or cooperation. Journal of Experimental Psychology: Human Perception and Performance, 8, 253-272.

Sergent, J., \& Lorber, E. (1983). Perceptual categorization in the cerebral hemispheres. Brain and Cognition, 2, 39-54.

Smith, E. E., \& Kemler, D. G. (1984). Overall similarity in adults' classification: The child in all of us. Journal of Experimental Psychology: General, 113(1), 137-159.

Smith, E. E., Patalano, A. L., \& Jonides, J. (1998). Alternative strategies of categorization. Cognition, 65, 167-196.

Sullivan, K. F., \& McKeever, W. F. (1985). The roles of stimulus repetition and hemispheric activation in visual half-field asymmetries. Brain and Cognition, 4, 413-429.

Tanaka, J., Luu, P., Weisbrod, M., \& Kiefer, M. (1999). Tracking the time course of object categorization using event-related potentials. Neuroreport, 10, 829-835.

Volberg, G., \& Hübner, R. (2004). On the role of response conflicts and stimulus position for hemispheric differences in global/local processing: An ERP study. Neuropsychologia, 42, 1805-1813.

Wyke, M., \& Ettlinger, G. (1961). Efficiency of recognition in left and right visual fields. Archives of Neurology, 5, 659-665. 